\title{
Repeated stimulation or tonic-signaling chimeric antigen receptors drive regulatory $T$ cell exhaustion
}

\section{Running Title: Exhaustion in human Tregs}

\author{
Caroline Lamarche ${ }^{1,2}$, German E. Novakovsky ${ }^{2,3}$, Christopher N. Qi ${ }^{1,2}$, Evan W. Weber ${ }^{4,5}$, \\ Crystal L. Mackall ${ }^{4,5}$, and Megan K Levings ${ }^{1,2,6}$
}

\author{
Author affiliations: \\ ${ }^{1}$ Department of Surgery, University of British Columbia, Vancouver, BC, Canada \\ ${ }^{2}$ BC Children's Hospital Research Institute, Vancouver, BC, Canada, \\ ${ }^{3}$ Department of Medical Genetics, University of British Columbia, Vancouver, BC, Canada \\ ${ }^{4}$ Department of Pediatrics and Medicine, Stanford University School of Medicine, Stanford, CA, \\ USA. \\ ${ }^{5}$ Stanford Cancer Institute, Stanford University School of Medicine, Stanford, CA, USA \\ ${ }^{6}$ School of Biomedical Engineering, University of British Columbia, Vancouver, BC, Canada
}

\section{Corresponding author:}

Megan Levings, mlevings@bcchr.ca

ORCID ID: 0000-0002-0305-5790

BC Children's Hospital Research Institute

A4-186, 950 West 28th Ave, Vancouver, BC, Canada V5Z 4H4 


\begin{abstract}
Regulatory T cell (Treg) therapy is a promising approach to improve outcomes in transplantation and autoimmunity. In conventional $\mathrm{T}$ cell therapy, chronic stimulation can result in poor in vivo function, a phenomenon termed exhaustion. Whether or not Tregs are also susceptible to exhaustion, and if so, if this would limit their therapeutic effect, was unknown. We studied how two methods which induce conventional T cell exhaustion - repetitive stimulation or expression of a tonic-signaling chimeric antigen receptor (CAR) - affect human Tregs. With each repetitive polyclonal stimulation Tregs progressively acquired an exhausted phenotype, and became less suppressive in vitro. Tregs expressing a tonic-signaling CAR rapidly acquired an exhausted phenotype and had major changes in their transcriptome and metabolism. Although tonicsignaling CAR-Tregs remained stable and suppressive in vitro, they lost in vivo function, as tested in a model of xenogeneic graft-versus-host disease. The finding that human Tregs are susceptible to exhaustion has important implications for the design of Treg adoptive immunotherapy strategies.
\end{abstract}




\section{Introduction}

Regulatory T cells (Tregs) control immune homeostasis and their adoptive transfer is a promising therapeutic approach with multiple trials completed, on-going or planned to test their efficiency in autoimmunity, solid organ transplantation or hematopoietic stem cell transplantation (reviewed in (Ferreira et al., 2019)). As Tregs are relatively rare, the majority of cell therapy protocols involve in vitro stimulation and expansion, in some cases culturing cells for up to 36 days to achieve $>2,000$ fold expansion (MacDonald et al., 2019b). Although these in vitro-expanded cells typically remain $\mathrm{FOXP}^{+}$and suppressive in vitro, whether or not repeated stimulation of Tregs affects their in vivo function is unknown.

In contrast to the paucity of knowledge regarding how chronic stimulation affects Treg function, the effects of chronic in vitro or in vivo stimulation of $\mathrm{T}$ cells has been extensively studied. For example, in the context of infection, cancer or transplantation, chronic exposure to antigen leads to a phenomenon termed exhaustion, characterized by high expression of inhibitory receptors such as programmed cell death protein 1 (PD-1), T-cell immunoglobulin and mucindomain containing-3 (TIM-3), and lymphocyte-activation gene 3 (LAG-3), diminished proliferative and cytokine-producing capacity, and high apoptosis (Fribourg et al., 2019; Saeidi et al., 2018; Thorp et al., 2015; Wherry and Kurachi, 2015). Although most research in this area examined effects on $\mathrm{CD} 8^{+} \mathrm{T}$ cells or unfractionated $\mathrm{CD} 3^{+} \mathrm{T}$ cells, but there is evidence that $\mathrm{CD}^{+} \mathrm{T}$ cells are similarly susceptible to exhaustion (Lynn et al., 2019; Shahbaz et al., 2020; Zou et al., 2020).

Exhaustion can also be induced by expression of engineered receptors, including chimeric antigen receptors (CARs), which mediate tonic signaling via spontaneous aggregation in the absence of antigen (Long et al., 2015; Lynn et al., 2019; Weber et al., 2020b). CAR-mediated T 
cell exhaustion induces expression of a variety of inhibitory receptors and diminishes their antitumor therapeutic efficacy (Finney et al., 2019; Wherry and Kurachi, 2015). This state of functional exhaustion can be manipulated by limiting activity of signaling pathways, and altering the epigenetic program and/or metabolic pathways (Chen et al., 2019; Fraietta et al., 2018; Lynn et al., 2019; Weber et al., 2020b).

Whether or not Tregs are also susceptible to exhaustion has not been explored. Here we used two different methods to chronically stimulate human Tregs and investigated the phenotypic, metabolic and functional consequences.

\section{Results and Discussion}

\section{Repetitive polyclonal Treg stimulation leads to decreased suppressive function}

Tregs are relatively rare cells that are hypo proliferative, so most protocols to expand Tregs for adoptive immunotherapy typically involve at least two polyclonal stimulations with anti-CD3 and -CD28 mAbs to obtain clinically relevant numbers (MacDonald et al., 2019b). To ask if repetitive stimulation could be detrimental for Treg function, we polyclonally stimulated Tregs weekly for 4 weeks and analyzed changes in their phenotype and function over time. Experiments were done with naïve $\mathrm{CD} 4{ }^{+} \mathrm{CD} 25^{\text {hi }} \mathrm{CD} 27^{\text {low }} \mathrm{CD} 45 \mathrm{RA}^{+}$Tregs (Supp Figure 1A) to minimize contamination with $\mathrm{CD}^{+}$conventional $\mathrm{T}$ cells (Tconvs) and/or unstable Tregs (Hoffmann et al., 2006). We first examined expression of cell surface receptors associated with exhausted T cells (Wherry and Kurachi, 2015), and found repeated Treg stimulation resulted in progressively higher expression of LAG-3 and TIM-3, but not of PD-1 (Figure 1A) or glucocorticoid-induced TNFR-related protein (GITR) (Figure 1B). 
We then measured cell-surface proteins associated with Treg function, finding that the naturally high expression of cytotoxic T-lymphocyte-associated protein 4 (CTLA-4) was not further enhanced by repeated stimulation. There was also no effect on expression of latencyassociated peptide (LAP, inactive TGF- $\beta$ ), or glycoprotein A repetitions predominant (GARP, a receptor for LAP) (Figure 1C). However, as seen with exhausted T cells (Wherry, 2011), repeatedly-stimulated Tregs became progressively less proliferative (decrease in $\% \mathrm{Ki} 7^{+}$cells) and viable (Figure 1D\&E).

To ask if these changes could be related to changes in Treg stability, we measured methylation at the Treg-specific demethylation region of FOXP3, finding no significant increase over time (Figure 1F). Similarly, FOXP3 expression was stable until the final re-stimulation when the average proportion of $\mathrm{FOXP}^{+}$cells declined to $75 \pm 5 \%$ at day 28 (Figure 1G). Consistent with preserved Treg stability, production of inflammatory cytokines remained low (Figure 1H). Nevertheless, as early as the second stimulation, the Tregs became progressively less suppressive as judged by the significant decrease in their ability to suppress division of $\mathrm{CD}^{+}$or $\mathrm{CD}^{+} \mathrm{T}$ cells (Figure 1I). Overall, these data indicate that repetitive, chronic stimulation of Tregs causes increased expression of some proteins characteristic of exhausted T cells and is deleterious for their in vitro function.

\section{Tonic signaling CARs induce a phenotype consistent with exhaustion in Tregs}

To further study the existence and biological relevance of Treg exhaustion, we next investigated the effect of expressing a tonic signaling CAR (TS-CAR), which incorporates a high-affinity scFv specific for disialoganglioside (GD2) fused to a CD28 co-stimulatory endodomain and

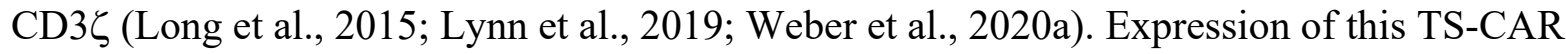
induces hallmark functional, phenotypic, transcriptional, and epigenetic features of exhaustion in 
T cells in vitro and abrogates antitumor effects in vivo (Long et al., 2015; Lynn et al., 2019).

Naïve $C D 4{ }^{+} \mathrm{CD} 127^{\text {lo }} \mathrm{CD} 25^{\text {hi }} \mathrm{CD} 45 \mathrm{RA}^{+} \mathrm{CD} 62 \mathrm{~L}^{\text {hi }}$ Tregs, or as a control naïve $\mathrm{CD} 4^{+}$

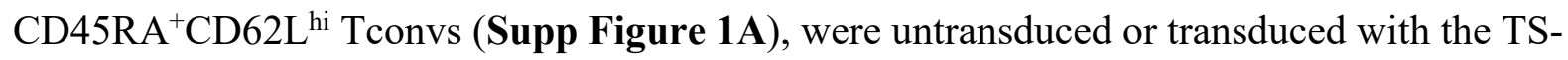

CAR, or a CD19-specific control CAR (non-TS-CAR) which neither mediates tonic signaling nor induces exhaustion (Long et al., 2015; Lynn et al., 2019). After 7 days, CAR-expressing cells were sorted then cultured for an additional 5 days before analysis. Phenotypic analysis revealed that, in comparison to untransduced or non-TS-CAR Tregs, a significantly higher proportion of TS-CAR Tregs expressed inhibitory receptors associated with exhaustion, namely LAG-3, TIM3, PD-1 (Figure 2A and Supp Figure 1B) as well as GITR (Figure 2B and Supp Figure 1B). Similarly, a higher proportion of TS-CAR Tregs expressed the Treg-function-associated proteins CTLA-4, LAP and GARP (Figure 2C and Supp Figure 1B). High expression of all these proteins was also observed in TS-CAR-expressing $\mathrm{CD}^{+}{ }^{+}$Tconvs and $\mathrm{CD} 8^{+} \mathrm{T}$ cells (Supp Figure 2A). Contrary to our observations with repeatedly stimulated Tregs, a higher proportion of TSCAR Tregs and $\mathrm{CD}^{+}$Tconvs were $\mathrm{Ki}^{+} 7^{+}$(Figure 2D and Supp Figure 2B), which could be indicative of a high proportion of terminally exhausted cells as opposed to progenitor exhausted cells (Miller et al., 2019). Although viability was similar between the 3 groups, in accordance with exhausted T cells (Zhang et al., 2020), TS-CAR Tregs were more apoptotic than controls (Figure 2E).

These phenotypic changes were not associated with loss of Treg stability, since TS-CAR Tregs had no change in TSDR methylation and actually increased FOXP3 expression (Figure 2 F\&G). Nevertheless, compared to controls, a significantly higher proportion of TS-CAR Tregs spontaneously produced IL-2 (Figure 2H), suggesting that these cells overcame FOXP3mediated repression of IL2 transcription (Bettelli et al., 2005). Overall, these data show that 
Tregs expressing a TS-CAR acquire a phenotype which mirrors that acquired by exhausted CD $8^{+}$ T cells and CD4 ${ }^{+}$Tconvs (Supp Figure 2) (Lynn et al., 2019).

\section{Transcriptome analysis of tonic signaling CAR-Tregs reveals changes in metabolic pathways}

A limitation of studying exhaustion in Tregs is that several of the canonical exhaustion makers are also markers of Tregs, or simply of activated T cells (Tanaka and Sakaguchi, 2019; Wherry and Kurachi, 2015). Moreover, since Tregs do not produce inflammatory cytokines, loss of cytokine production cannot be monitored as a surrogate marker of dysfunction. Therefore, we next took an unbiased approach and used RNA sequencing to determine how tonic signaling affected the Treg transcriptome in comparison to untransduced or non-TS-CAR Tregs, or CD4 ${ }^{+}$ Tconvs. Multi-dimensional scaling plots revealed that Tregs and Tconvs clustered separately, and that, for both Tregs and Tconvs, the TS-CARs were further away from untransduced cells than cells expressing the non-TS-CAR cells (Figure 3A).

To better understand the consequences of exhaustion in Tregs, we first sought to identify differences in gene expression between TS-CAR Tregs and TS-CAR CD4 ${ }^{+}$Tconvs. As expected, in comparison to TS-CAR CD4 ${ }^{+}$Tconvs, TS-CAR Tregs had low expression of effector molecules such as GZMA, GZMB (granzyme A \&B), IFNG, TNFSF10 (TRAIL) and FASLG, and high expression of Treg-related genes such as FOXP3, IKZF2 (Helios) and LRRC32 (GARP), confirming their Treg identity (Figure 3B). Interestingly, inhibitory surface receptors were differentially expressed in TS-CAR Tregs compared to TS-CAR CD4 ${ }^{+}$Tconvs, with lower expression of CD244, PDCD1 (PD1) and LAG3 and higher expression of TIGIT and CTLA4. Similarly, there were differences in expression of exhaustion-related transcription factors (Zhang 
et al., 2020), with higher expression of TOX, but not EOMES or TBX21 in TS-CAR Tregs compared to TS-CAR CD4 ${ }^{+} \mathrm{T}$ cells (Figure 3B).

Since many of the genes differentially expressed between TS-CAR Tregs and Tconvs were simply characteristic of known transcriptional differences between these cell lineages, we also examined differential gene expression after removing genes that were differentially expressed between UT Tregs and UT Tconvs (Figure 3C and Supp Figure 3A). The resulting data revealed that compared to $\mathrm{CD} 4^{+}$TS-CAR Tconvs, TS-CAR Tregs expressed significantly less $B A T F 3$, a transcription factor expressed in exhausted TS-CAR T cells (Lynn et al., 2019; Weber et al., 2020b) and less CD244, an immunoregulatory receptor also associated with T cell exhaustion (Agresta et al., 2018; Schlaphoff et al., 2011). TS-CAR Tconvs also expressed more chemokines (CCL1, CXCL1 and CXCL8), cytokines (IL21, IL22 and LIF) and integrin $\alpha 1$ (ITGA1). On the other hand, TS-CAR Tregs overexpressed GPA33, the expression of which is a marker of stable Tregs (Opstelten et al., 2020), and GARP (LLRC32), confirming the notion that tonic signaling in Tregs does not impair their lineage stability.

Within the 3 groups of Tregs, the transcriptome of the TS-CAR Tregs was clearly distinct from UT and non-TS-CAR Tregs, with a heat map of the 50 most differentially expressed genes between these groups shown in Figure 3D. In comparison to both UT and non-TS CAR Tregs, TS-CAR Tregs had high expression of NR4A3 (NOR1) and NR4A1 (NUR77), two genes associated with exhaustion in $\mathrm{CD}^{+}$T cells (Chen et al., 2019; Liu et al., 2019). TS-CAR Tregs also had lower RICTOR, which is part of the mammalian target of rapamycin (mTOR) complex 2 (mTORC2) and implicated in control of Treg suppressive function (Charbonnier et al., 2019). There was also a decrease in $R U N X 2$, the expression of which is enhanced by a weak rather than a strong TCR stimulation (Olesin et al., 2018). Confirming the flow cytometry data, TS-CAR 
Tregs had high expression of exhaustion-related genes such as PDCD1 (PD1), HAVCR2 (TIM3),

TIGIT, LAG3 and CTLA4, but low expression of several transcription factors known to be downregulated in terminally exhausted T cells, including BCL6, SATB1, TCF7 (which encodes TCF-1), LEF1 and BACH2 (Blank et al., 2019; Miller et al., 2019; Wang et al., 2019) (Figure 3E).

Hallmark pathway analysis, obtained by Gene Set Enrichment Analysis, revealed that TSCAR Tregs upregulated pathways linked to metabolism, including MYC and MTORC1 signaling, glycolysis, oxidative phosphorylation, cholesterol homeostasis, fatty acid metabolism and adipogenesis (Figure 3F), suggesting that changes in cell metabolism may be key factors driving the observed phenotypic changes in TS-CAR Tregs. However, although these pathways were upregulated in TS-CAR Tregs compared to other Treg groups, they were still lower in comparison to TS-CAR CD4 ${ }^{+}$Tconvs (Supp Figure 3B).

\section{Tonic signaling in Tregs causes an increase in glycolysis}

A growing body of evidence suggests that differences in metabolic pathway activity underlie functional differences between Tregs and Tconvs (Beier et al., 2015). Specifically, oxidative phosphorylation and mitochondrial activity, which are required for Treg suppressive function (Pacella and Piconese, 2019; Saravia et al., 2020), are promoted by FOXP3 via inhibition of cMyc-driven glycolysis (Angelin et al., 2017; Gerriets et al., 2016; Howie et al., 2017; Wang et al., 2011). In the context of exhaustion, metabolic dysfunction is known to precede its development in T cells (McKinney and Smith, 2018), and is influenced by CAR expression (Kawalekar et al., 2016). Although FOXP3 expression was similar between UT, non-TS- and TS-CAR Tregs, transcriptomic analysis suggested that there could be TS-CAR related changes in 
metabolic activity. Therefore, we analyzed their extracellular acidification rate (ECAR) and oxygen consumption rate (OCR) and as surrogate measures of glycolysis and oxidative phosphorylation, respectively. In their basal state TS-CAR Tregs had a tendency to have higher ECAR and OCR than UT or non-TS-CAR Tregs, suggesting both metabolic pathways were enhanced (Figure 4A\&B, left). However, a significantly lower relative OCR/ECAR ratio showed that TS-CAR Tregs preferentially engaged glycolysis compared to their UT or non-TSCAR counterparts (Figure 4C).

To further interrogate these pathways, cells were exposed to oligomycin, a mitochondrial ATP synthase inhibitor which prevents oxidative phosphorylation and usually upregulates glycolysis. Since TS-CAR Tregs had high basal ECAR, the relative oligomycin-stimulated increase was modest compared to the other groups, suggesting limited spare glycolytic capacity (Figure 4A). In contrast there was comparable ATP-coupled mitochondrial respiration in all groups (Figure 4B). Injection of carbonyl cyanide-4 (trigluoromethoxy) phenylhydrazone (FCCP) to maximize mitochondrial oxygen consumption, revealed that TS-CAR Tregs also had lower spare respiratory capacity (Figure 4D). Data in Tregs were mirrored with CD4 ${ }^{+}$Tconvs (Supp Figure 3C). Overall these data suggest that TS-CAR Tregs would poorly adapt to an increase in energy demand due to their high basal activity of both of these pathways (Schurich et al., 2016; van der Windt et al., 2012).

\section{TS-CAR Tregs are dysfunctional in vivo but not in vitro}

In mice upregulation of glycolysis and/or inhibition of oxidative phosphorylation leads to reduced Foxp3 expression and/or suppressive function (Beier et al., 2015; Gerriets et al., 2016; Wei et al., 2016). We therefore next asked how TS-CAR expression affected Treg function. We 
first tested suppressive function in vitro by co-culturing increasing numbers of UT, non-TS-, or TS-CAR Tregs with anti-CD3/28-stimulated PBMCs and measuring proliferation after 4 days. Unexpectedly, TS-CAR Tregs were significantly more potent than UT or non-TS Tregs at suppressing $\mathrm{CD}^{+}$and $\mathrm{CD}^{+} \mathrm{T}$ cell proliferation (Figure 5A), but within the Treg population, the TS-CAR Tregs proliferated significantly less than UT or non-TS-CAR Tregs (Figure 5B). The increase in TS-CAR Tregs suppressive function despite their lower division index could be due to a higher suppression potential on a per cell basis and/or by the immunomodulatory potential of apoptotic Tregs (Maj et al., 2017).

To address why expression of a TS-CAR increased, whereas repetitive polyclonal stimulation decreased (Figure 1H) in vitro suppression, we noted the difference in PD-1 expression. Specifically, PD-1 was highly expressed by TS-CAR Tregs but not by repetitively stimulated Tregs. To investigate if the increased in vitro suppressive activity of TS-CAR Tregs was mediated by PD-1, suppression assays were repeated in the presence of nivolumab, an inhibitory anti-PD-1 mAb. Although nivolumab did not reverse the heightened suppressive activity of TS-CAR Tregs (Figure 5C), it reversed the decreased TS-CAR Treg-intrinsic proliferation (Figure 5D). The beneficial effect of PD-1 inhibition on Treg proliferation is consistent with the finding that PD-1 blockade in patients can sometimes lead to paradoxical cancer progression due to enhanced Treg activity (Kamada et al., 2019).

Studies of TS-CARs in Tconvs showed that despite normal in vitro effector activity, functional defects were revealed in vivo (Long et al., 2015) so we next tested Treg function in vivo using a humanized mouse model of xenogeneic graft-versus-host disease. NSG mice were injected with $6 \times 10^{6}$ PBMCs without or with $3 \times 10^{6}$ UT or TS-CAR (n.b. non-TS Tregs not examined as they would be stimulated by $\mathrm{CD} 19^{+} \mathrm{B}$ cells in the PBMCs), monitored and bled 
weekly (Figure 5E). Surprisingly, TS-CAR Tregs completely lost their in vivo protective effect, with no improved survival in comparison to mice which only received PBMC. In contrast, mice which received UT Tregs were protected from mortality over this time period (Figure 5F). Human cell engraftment mirrored the clinical effect, with only the UT Tregs able to reduce the number of circulating and splenic human $\mathrm{CD} 45^{+}$cells in comparison to mice that only received PBMCs (Figure 5G).

Continuous TCR signaling is essential for maintenance of immune tolerance by Tregs (Levine et al., 2014; Vahl et al., 2014), but whether or not Tregs become susceptible to exhaustion with prolonged, high level levels of stimulation was an outstanding question. We used two clinically-relevant approaches to ask whether exhaustion has the potential to limit the therapeutic efficacy of Tregs. With both repetitive polyclonal stimulation and TS-CAR expression we found clear evidence for development of a functional deficit consistent with the concept of exhaustion. Whereas repeated polyclonal stimulation gradually decreased in vitro suppression over time, expression of a TS-CAR caused rapid phenotypic and functional changes, and complete loss of suppression function in vivo. Importantly, loss of suppression function was not correlated with loss of Treg lineage stability, indicating that undesired acquisition of effector function is not a sequalae of chronic stimulation. These findings have important implications for the design of Treg adoptive transfer therapies and highlight the need to assess suppressive function both in vitro and in vivo.

A challenge to studying Treg exhaustion in vitro is the fact that inhibitory receptors characteristic of exhausted Tconvs are often associated with enhanced Treg suppressive potential (Anderson et al., 2016; Gautron et al., 2014; Gianchecchi and Fierabracci, 2018). For example, CTLA4 is essential for Treg function (Wing et al., 2008) and TIM-3 ${ }^{+}$Tregs are more potent than 
their TIM-3- counterparts (Gautron et al., 2014). Moreover, as Tregs do not normally secrete inflammatory cytokines or exhibit cytolytic activity, in vitro analysis of these activities cannot be used to gauge this phenomenon. Indeed, repeated stimulation and TS-CAR expression increased expression of LAG-3 and TIM-3 but had little effect on other classical surface markers of exhaustion. Since cytometry-based methods typically used to evaluate exhaustion in T cells seem to have limited relevance in Tregs, it will of interest to study if cell surface proteins upregulated in TS-CAR Tregs at the mRNA level, including FCER2 (CD32), IL1R2 (IL1 decoy receptor), CD276 (B7-H3) and ITGB8 (integrin beta 8), could be used to monitor this state.

Gene expression analysis revealed high expression of exhaustion-related transcription factors, including $N R 4 A 1$ and 3, BATF and PRDM1 (Blimp1), suggesting there is at least some overlap with the transcriptional program that induces exhaustion in Tregs and in T cells. Moreover, as with exhausted T cells (Kawalekar et al., 2016; Wherry, 2011), exhausted Tregs displayed increased apoptosis, and underwent metabolic remodeling that reduced metabolic flexibility (Schurich et al., 2016). Poor metabolic flexibility in exhausted Tregs could underlie the difference in function in vitro versus in vivo: in comparison to in vitro cultures with saturated metabolite availability, limited availability of metabolic substrates in vivo could contribute to the profound loss of TS-CAR Treg function in vivo.

In mice, Tregs with a liver kinase B1 (Lkb1) deletion expressed a similar phenotype to TS-CAR Tregs with high expression of Pd1 and Gitr, increased apoptosis, and altered metabolism (Yang et al., 2017). Notably, Lkb1-deficient Tregs failed to suppress Th2 responses in vivo, an effect reversed by PD1 blockade (Yang et al., 2017). Although we did not observe decreased expression of $L K B 1$, or of other genes found to mediate the "exhausted" phenotype in mouse Lkb1-deficient Tregs, including CTNNB1 $\beta$-catenin and HDC (histidine decarboxylase), 
this pathway could still be affected due to alterations in downstream kinase mediators, and/or post transcriptional effects on LKB kinase activity. Investigation into how Treg exhaustion in humans might relate to this pathway, specifically with respect to effects on defined Th cell subsets, will be an important area of future investigation.

We noted similarities and differences between Tregs subject to chronic re-stimulation versus activity of a TS-CAR. In both cases there was increased expression of LAG-3 and TIM-3 and functional manifestations, but differential effects on proliferation, FOXP3 expression and in vitro suppressive function. The latter maybe driven by high expression of inhibitory receptors other than PD-1 by the TS-CAR Tregs. A better understanding of how such receptors contribute to Treg function in the context of exhaustion is important for understanding how immune checkpoint inhibitors might affect immune suppression in cancer and autoimmunity (Saleh and Elkord, 2019).

Finding ways to mitigate Treg exhaustion is critical for the continued development of successful Treg adoptive therapy. Our data highlight that repetitive stimulation should be limited during cell manufacturing, and that for optimal function, engineered receptors should be designed to limit antigen-independent activity. Our data also highlight to need to assess suppressive function in vivo, as similar to exhausted T cells, the design of typical in vitro assays may not fully reveal functional defects. An open question is how repeated exposure of a Treg to its target in vivo would affect long-term function. For example, even in the absence of tonic signaling, how continuous presence of an auto or alloantigen would affect a CAR- or TCRengineered Tregs will be an important consideration. The metabolic phenotype of exhausted Tregs suggests that, as for $\mathrm{CD}^{+} \mathrm{T}$ cells and $\mathrm{CD}^{+} \mathrm{T}$ convs, strategies to modulate signaling pathway activity for example by use of kinase inhibitors (Dufva et al., 2020; Mestermann et al., 
2019; Weber et al., 2019; Weber et al., 2020b), or modulation of transcription factor expression (Chen et al., 2019; Lynn et al., 2019), could be strategies to limit this risk.

\section{Material and Methods}

T cell sorting. $\mathrm{CD}^{+} \mathrm{T}$ cells were isolated from healthy adults using the RosetteSep human $\mathrm{CD} 4^{+}$ T cell enrichment cocktail (STEMCELL Technologies, 15062) followed by a density gradient isolation (STEMCELL Technologies, 07861). Naïve Tregs were sorted from a CD25 enriched fraction ((Miltenyi, 130-092-983) $\mathrm{CD}^{+} \mathrm{CD} 127^{\mathrm{lo}} \mathrm{CD} 25^{\mathrm{hi}} \mathrm{CD} 45 \mathrm{RA}^{+} \mathrm{CD} 62 \mathrm{~L}^{\mathrm{hi}}$ cells and naïve CD4 ${ }^{+}$ Tconvs were sorted from the CD25 negative fraction as live $\mathrm{CD}^{+} \mathrm{CD}^{+} \mathrm{RA} \mathrm{A}^{+} \mathrm{CD} 62 \mathrm{~L}^{\text {hi }}$ cells using a MoFlo ${ }^{\circledR}$ Astrios (Beckman Coulter). Cells were resuspended at $1 \times 10^{6}$ cells $/ \mathrm{ml}$ in XH media (X-Vivo 15 (Lonza) with 5\% human serum, 1\% penicillin/streptavidin, 1\% glutamine and phenol red) containing 300U IL2/ml (Proleukin).

Repetitive stimulation: sorted naive Tregs and Tconvs were stimulated with artificial antigen presenting cells (MacDonald et al., 2019a) loaded with aCD3 mAb (OKT3, UBC AbLab; 100ng/mL) in XH media with 300U IL2/ml, or stimulated with CD3/28 Dynabeads (ThermoFisher Scientific, 11141D) 3 bead : 1 cell ratio. Every week, a portion of cells was used for assays and the remaining were re-stimulated using the same initial stimulus with the exception that the Dynabead ratio was reduced to a 1 bead:1 cell ratio.

Viral vectors and transduction: MSGV retroviral vectors and retroviral supernatant encoding the CD19-28z and gd2-28z CARs were produced as previously described (Lynn et al., 2019). For the retroviral transduction, sorted cells were stimulated with a 3 bead: 1 cell ratio of Dynabeads in 
$\mathrm{XH}$ media containing 300U IL2/ml (Proleukin) as above. On the following day, non-tissue culture treated 96-well plates were coated overnight at $4{ }^{\circ} \mathrm{C}$ with $1 \mathrm{ml}$ of retronectin (Takara) at $25 \mu \mathrm{g} / \mathrm{ml}$ in PBS. On day 2 and 3, plates containing retronectin were washed with PBS and blocked with $2 \% \mathrm{BSA}$ for $15 \mathrm{~min} .100 \mu \mathrm{l}$ of thawed retroviral supernatant was then added to the well and centrifuged for $2 \mathrm{~h}$ at $32^{\circ} \mathrm{C}$ at $3,200 \mathrm{rpm}$ before the addition of the same volume of media containing the activated cells. Media was refreshed on day 5. At day 7, cells were collected and stained with a fixable viability dye (FVD, Thermo Fisher Scientific), CD4 (clone RPA-T4, BD) and an anti-14g2a idiotype antibody (clone 1A7, NIH) or Protein L (genscript) to sort cells expressing the GD2- or CD19-specific CARs, respectively. Live $\mathrm{CD}^{+} \mathrm{CAR}^{+}$cells or live $\mathrm{CD}^{+}$cells (for the untransduced controls) were sorted (MoFlo® Astrios) resuspended at $0.5 \times 10^{6}$ cells $/ \mathrm{ml}$ in XH media containing $300 \mathrm{U}$ IL2 $/ \mathrm{ml}$ for an addition $4-5$ days. Throughout cell culture, cell number and viability were assessed using the Cellometer auto2000 and an AOPI Staining solution (Nexelcom Bioscience).

Flow cytometry. Cells were stained with fixable viability dye (FVD, Thermo Fisher Scientific, 65-0865-14) and surface markers in PBS for $30 \mathrm{~min}$ at $4^{\circ} \mathrm{C}$ before fixation and permeabilization using eBioscience FOXP3/Transcription Factor Staining Buffer Set (Thermo Fisher Scientific, 00-5523-00) and staining for intracellular proteins. Samples were analyzed on a BD LSR II (BD Bioscience) or a Cytoflex (Beckman Coulter) and results analyzed using FlowJo Software version 10.5.3. We used a FVD dye (Thermo Fisher Scientific, 65-0865-14), and the following antibodies from BD Biosciences; CD4 (RPA-T4), GARP (7B11), Ki67 (B56), CTLA4 (BNI3), CD8 (HIT8a), CD3 (UCHT1), IL-2 (MQ1-17H12) and an APC-Streptavidin conjugated antibody (554067). We also used CD25 (4E3, Miltenyi), and the following antibodies from eBioscience; 
CD127 (eBioRDR5), CD45RA (HI100), CD62L (DREG-56), FOXP3 (236A/E7), LAG-3

(3DS223H), CD4 (RPA-T4) and TNF- $\alpha$ (MAb11), IL-17A (eBio64DEC17). CD8 (RPA-T8),

TIM-3 (F38-2E2), PD-1 (EH12.2H7), CD69 (FN50), IFN- $\gamma$ (4S.B3) and Helios (22F6) were

from Biolegend and GITR (110416) and LAP (27232) from R\&D.

Apoptosis was assessed using the apoptosis/necrosis detection kit (Abcam, ab176749) according to the manufacturer's instructions. For intracellular cytokine staining, $0.1 \times 10^{6}$ cells were activated with PMA $(10 \mathrm{ng} / \mathrm{ml})$ and ionomycin $(500 \mathrm{ng} / \mathrm{ml})$ in the presence of Brefeldin A $(10 \mu \mathrm{g} / \mathrm{ml})$ for $4 \mathrm{~h}$ at $37^{\circ} \mathrm{C}$. Surface and intracellular staining was done as described above.

To monitor human cell engraftment in mice, $50 \mu \mathrm{L}$ of blood was collected weekly and at

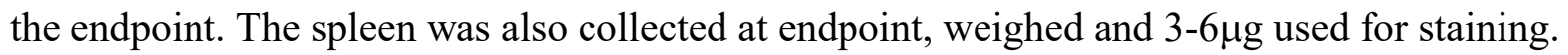
Red blood cells were lysed with ammonium chloride and remaining cells were resuspended in PBS containing anti-mouse CD16/32 (Thermo Fisher Scientific, 14-0161-82) for 10 minutes and then stained for extracellular markers using fixable viability dye (FVD; Thermo Fisher

Scientific, 65-0865-14), anti-mouse CD45 (Thermo Fisher Scientific, 25-0451-82), anti-human CD45 (BD Biosciences, HI30), CD4 (Biolegend, RPA-T4), CD8 (eBioscience, HIT8a) as previously described (Dawson et al., 2019). Ten thousand counting beads were added to every sample (Thermo Fisher Scientific, 01-1234-42).

TSDR. DNA was isolated and bisulfite-converted using the EZ DNA Methylation-Direct Kit (Cedarlane labs, D5021). A PCR of BisDNA was then made using the folowing primers: AGAAATTTGTGGGGTGGGGTAT (PCR FWD), ATCTACATCTAAACCCTATTATCACAACC (PCR REV-Bio) and AGAAATTTGTGGGGTGGG (SEQ FWD), followed by pyrosequencing using PyroMark 
buffers and assay on a Biotage PyroMark Q96 MD pyrosequencer (Qiagen). Methylation of the TSDR were calculated with the Pyro Q-CpG software (Biotage). Average methylation of the CpG sites 49260827, 49260817, 49260814, 49260808, 49260804, 49260796, 49260787 according to their position on the X chromosome (reference genome GRCh38) were reported.

Seahorse Extracellular Flux assay. Seahorse XF Cell mito stress test assays were done using a Seahorse XFe96 Extracellular Flux Analyzer (Agilent) according to the manufacturer's instructions. The day before, cartridge was hydrated using sterile water. The plate was coated with Cell-Tak solution (BD) and both the plate and $25 \mathrm{ml}$ of XF calibrant (Agilent) were placed overnight at $37^{\circ} \mathrm{C}$ in a non-CO2 incubator. On the day of the assay, Cell-Tak was removed, washed with PBS and cells were counted and resuspended in Seahorse XF RPMI media, pH 7.4 (Agilent), supplemented with 1\% FBS, $1 \mathrm{mM}$ glutamax and $10 \mathrm{mM}$ glucose. $2 \times 10^{5}$ live cells/well were plated in triplicate when possible. Oligomycin $(1 \mu \mathrm{M}), \mathrm{FCCP}(1.5 \mu \mathrm{M})$ and Rotenone/Antimycin A (1 $\mu \mathrm{M})$ (all Sigma) were added as indicated in port A, B and C.

Suppression assay. Allogeneic PBMCs were labeled with ef450 and Tregs with ef670 (ThermoFischer Scientific, 65-0842 and 65-0840, respectively). 0.1x106 PBMCs were then plated in a 96-well plate with CD3/28 Dynabeads at a 1:16 cell: bead ratio. Labeled Tregs were added at the indicated ratios. For some experiments, nivolumab (Bristol Myers Squibb) was added at $10 \mu \mathrm{g} / \mathrm{ml}$. After 4 days, cells were stained extracellularly and CD4 ${ }^{+}, \mathrm{CD}^{+}$and Treg proliferation was assess by flow cytometry. Suppression was determined as 100-(Division index with Tregs/Division index without Tregs *100). 
Xenogeneic graft-versus-host disease. 8- to 12-week-old male and female NSG mice (NOD.Cg$\operatorname{Prkdc^{scid}} \mathrm{Il2} \mathrm{rg}^{t m 1} \mathrm{Wjl} / \mathrm{SzJ}$, The Jackson Laboratory, bred in house) received whole-body irradiation (150 cGy, RS-2000 Pro Biological System), and one day later i.v. injected with PBS or 6 x $10^{6}$ PBMCs in the absence or presence of $3 \times 10^{6}$ allogeneic untransduced or TS-CAR Tregs. Graft versus host disease was scored on the basis of weight, fur texture, posture, activity level, and skin integrity, with 0 to 3 points per category as described (Dawson et al., 2019). Mice were euthanized during the course of the experiment if they had a total score of 6 or a score of 3 in any category. Flow cytometric analysis to measure human immune cell engraftment is described above.

RNA sequencing: RNA was extracted from $0.25 \times 10^{6}$ cells at day 12 of cell culture using the Monarch total RNA miniprep kit (New England Biolabs, T2010S) and quantified using the Qubit RNA HS Assay kit (Thermo Fisher Scientific, Q32855) according to the manufacturer's instructions. RNA purity (RNA Integrity Number) was assessed using the RNA 6000 Pico assay (Agilent, 5067-1513) and samples prepared using the TruSeq stranded mRNA library kit (Illumina) on the Illumina Neoprep automated nanofluidic prep instrument. Illumina NextSeq 500 with Paired End $42 \mathrm{bp} \times 42 \mathrm{bp}$ reads was used for sequencing and de-multiplexed read sequences and sequenced were then aligned to the Homo sapiens (PAR-masked)/hg19 reference using STAR aligner (version 2.5.0a) and the RNA-Seq Alignment App (version 1.1.0) on Illumina Basespace. De-multiplexed read sequences and RnaReadCounter (part of the internal analysis tool IsisRNA (version 2.6.25.18)) was used for counting the number of aligned reads, as described previously (Hoeppli et al., 2019). In R, raw count matrices were generated using HTSeq (v0.11.2), then scale factors were calculated to take into account differences in library 
sizes using edgeR (v3.24.3) and normalization was performed using limma (v3.38.3) as in (Law et al., 2016). Log (CPM) and visualization was performed using: ggplot2 (3.2.1), RColorBrewer (v1.1.2), tibble (2.1.3), pheatmap (v1.0.12), stats (v3.5.1), and gplots (v3.0.1.2). The Gene Set Enrichment Analysis was performed with fgsea (v1.8.0) and Hallmark gene sets from MSigDB (v6.2). The code used for data analysis is available on GitHub:

https://github.com/fransilvion/RNA_seq_Lamarche. RNAsequencing data are deposited on NCBI GEO, GSE153384.

Statistical analysis. All statistics were done using Prism version 8.4.2 and are described in the figure legends. For the experiments where multiple assays were repeated over time (Figure 1), one-way ANOVA or mixed-effects analysis with Dunnett's multiple comparisons test were used, comparing each data to their matched results at day 7. To compare the different Tregs, one-way Anova with Turkey's comparisons test were done. **** $\mathrm{p} \leq 0.0001, * * * \mathrm{p} \leq 0.001, * * \mathrm{p} \leq 0.01$, $* \mathrm{p} \leq 0.05$.

Study Approval. Healthy volunteers gave written informed consent to use their blood according to protocols approved by the University of British Columbia Clinical Research Ethics Board (UBC-CREB) and Canadian Blood Services. Animal protocols were approved by the UBC Animal Care Committee. 


\section{Author contributions}

CL conceived, designed and performed experiments, analyzed data and wrote the manuscript;

GEN carried out bioinformatic analysis and critically reviewed the manuscript; CNQ conducted experiments and analyzed data; EWW and CLM provided essential reagents, intellectual input and critically reviewed the manuscript; MKL conceived and designed experiments, provided overall direction and interpretation, obtained funding and wrote the manuscript.

\section{Acknowledgments}

This work was supported by a grant from the Canadian Institutes of Health Research (CIHR) FDN-154304. CL is supported by a CIHR fellowship and a KRESCENT fellowship award. MKL receives a salary awards from the BC Children's Hospital Research Institute. We thank Dr. Ramon Klein-Geltnick for advice and critical reading of the manuscript and Dr. Lixin Xu for expert flow cytometry support. MKL received research funding from TxCell, Bristol-Myers Squibb, Pfizer, Takeda and CRISPR Therapeutics for work unrelated to this report. CLM holds several patent applications in the area of CAR based immunotherapy, and is a founder of, holds equity in, and receives consulting fees from Lyell Immunopharma which develops CAR based therapies. EWW holds several patent applications in the area of CAR based immunotherapy and receives consulting fees from Lyell Immunopharma. CLM is a member of the Parker Institute for Cancer Immunotherapy, which supports the Stanford University Cancer Immunotherapy Program. The authors have no additional financial interests. 


\section{Abbreviations}

CAR, Chimeric antigen receptor

ECAR, extracellular acidification rate

OCAR, oxygen consumption rate

Tconv, conventional T cell

Treg, Regulatory T cell

TS, tonic signaling

TSDR, Regulatory T-cell-Specific Demethylated Region 


\section{References}

Agresta, L., K.H.N. Hoebe, and E.M. Janssen. 2018. The Emerging Role of CD244 Signaling in Immune Cells of the Tumor Microenvironment. Frontiers in immunology 9:2809.

Anderson, A.C., N. Joller, and V.K. Kuchroo. 2016. Lag-3, Tim-3, and TIGIT: Co-inhibitory Receptors with Specialized Functions in Immune Regulation. Immunity 44:989-1004.

Angelin, A., L. Gil-de-Gómez, S. Dahiya, J. Jiao, L. Guo, M.H. Levine, Z. Wang, W.J. Quinn, P.K. Kopinski, L. Wang, T. Akimova, Y. Liu, T.R. Bhatti, R. Han, B.L. Laskin, J.A. Baur, I.A. Blair, D.C. Wallace, W.W. Hancock, and U.H. Beier. 2017. Foxp3 Reprograms T Cell Metabolism to Function in Low-Glucose, High-Lactate Environments. Cell Metab 25:12821293.e1287.

Beier, U.H., A. Angelin, T. Akimova, L. Wang, Y. Liu, H. Xiao, M.A. Koike, S.A. Hancock, T.R. Bhatti, R. Han, J. Jiao, S.C. Veasey, C.A. Sims, J.A. Baur, D.C. Wallace, and W.W. Hancock. 2015. Essential role of mitochondrial energy metabolism in Foxp3 ${ }^{+}$T-regulatory cell function and allograft survival. FASEB journal 29:2315-2326.

Bettelli, E., M. Dastrange, and M. Oukka. 2005. Foxp3 interacts with nuclear factor of activated $\mathrm{T}$ cells and NF- $\mathrm{BB}$ to repress cytokine gene expression and effector functions of $\mathrm{T}$ helper cells. Proceedings of the National Academy of Sciences of the United States of America 102:51385143.

Blank, C.U., W.N. Haining, W. Held, P.G. Hogan, A. Kallies, E. Lugli, R.C. Lynn, M. Philip, A. Rao, N.P. Restifo, A. Schietinger, T.N. Schumacher, P.L. Schwartzberg, A.H. Sharpe, D.E. Speiser, E.J. Wherry, B.A. Youngblood, and D. Zehn. 2019. Defining 'T cell exhaustion'. Nature reviews. Immunology 19:665-674.

Charbonnier, L.-M., Y. Cui, E. Stephen-Victor, H. Harb, D. Lopez, J.J. Bleesing, M.I. GarciaLloret, K. Chen, A. Ozen, P. Carmeliet, M.O. Li, M. Pellegrini, and T.A. Chatila. 2019. Functional reprogramming of regulatory T cells in the absence of Foxp3. Nature immunology 20:1208-1219.

Chen, J., I.F. Lopez-Moyado, H. Seo, C.J. Lio, L.J. Hempleman, T. Sekiya, A. Yoshimura, J.P. Scott-Browne, and A. Rao. 2019. NR4A transcription factors limit CAR T cell function in solid tumours. Nature 567:530-534.

Dawson, N.A., C. Lamarche, R.E. Hoeppli, P. Bergqvist, V.C. Fung, E. McIver, Q. Huang, J. Gillies, M. Speck, P.C. Orban, J.W. Bush, M. Mojibian, and M.K. Levings. 2019. Systematic testing and specificity mapping of alloantigen-specific chimeric antigen receptors in regulatory $\mathrm{T}$ cells. JCI Insight 4: e123672.

Dufva, O., J. Koski, P. Maliniemi, A. Ianevski, J. Klievink, J. Leitner, P. Pölönen, H. Hohtari, K. Saeed, T. Hannunen, P. Ellonen, P. Steinberger, M. Kankainen, T. Aittokallio, M.A.I. Keränen, 
M. Korhonen, and S. Mustjoki. 2020. Integrated drug profiling and CRISPR screening identify essential pathways for CAR T-cell cytotoxicity. Blood 135:597-609.

Ferreira, L.M.R., Y.D. Muller, J.A. Bluestone, and Q. Tang. 2019. Next-generation regulatory T cell therapy. Nat Rev Drug Discov 18:749-769.

Finney, O.C., H.M. Brakke, S. Rawlings-Rhea, R. Hicks, D. Doolittle, M. Lopez, R.B. Futrell, R.J. Orentas, D. Li, R.A. Gardner, and M.C. Jensen. 2019. CD19 CAR T cell product and disease attributes predict leukemia remission durability. J Clin Invest 129:2123-2132.

Fraietta, J.A., C.L. Nobles, M.A. Sammons, S. Lundh, S.A. Carty, T.J. Reich, A.P. Cogdill, J.J.D. Morrissette, J.E. DeNizio, S. Reddy, Y. Hwang, M. Gohil, I. Kulikovskaya, F. Nazimuddin, M. Gupta, F. Chen, J.K. Everett, K.A. Alexander, E. Lin-Shiao, M.H. Gee, X. Liu, R.M. Young, D. Ambrose, Y. Wang, J. Xu, M.S. Jordan, K.T. Marcucci, B.L. Levine, K.C. Garcia, Y. Zhao, M. Kalos, D.L. Porter, R.M. Kohli, S.F. Lacey, S.L. Berger, F.D. Bushman, C.H. June, and J.J. Melenhorst. 2018. Disruption of TET2 promotes the therapeutic efficacy of CD19-targeted T cells. Nature 558:307-312.

Fribourg, M., L. Anderson, C. Fischman, C. Cantarelli, L. Perin, G. La Manna, A. Rahman, B.E. Burrell, P.S. Heeger, and P. Cravedi. 2019. T-cell exhaustion correlates with improved outcomes in kidney transplant recipients. Kidney international 96:436-449.

Gautron, A.S., M. Dominguez-Villar, M. de Marcken, and D.A. Hafler. 2014. Enhanced suppressor function of TIM-3+ FoxP3+ regulatory T cells. European journal of immunology 44:2703-2711.

Gerriets, V.A., R.J. Kishton, M.O. Johnson, S. Cohen, P.J. Siska, A.G. Nichols, M.O. Warmoes, A.A. de Cubas, N.J. MacIver, J.W. Locasale, L.A. Turka, A.D. Wells, and J.C. Rathmell. 2016. Foxp3 and Toll-like receptor signaling balance Treg cell anabolic metabolism for suppression. Nature immunology 17:1459-1466.

Gianchecchi, E., and A. Fierabracci. 2018. Inhibitory Receptors and Pathways of Lymphocytes: The Role of PD-1 in Treg Development and Their Involvement in Autoimmunity Onset and Cancer Progression. Frontiers in immunology 9:2374.

Hoeppli, R.E., K.N. MacDonald, P. Leclair, V.C.W. Fung, M. Mojibian, J. Gillies, S.M.R. Rahavi, A.I.M. Campbell, S.K. Gandhi, A.M. Pesenacker, G. Reid, C.J. Lim, and M.K. Levings. 2019. Tailoring the homing capacity of human Tregs for directed migration to sites of Th1inflammation or intestinal regions. American journal of transplantation 19:62-76.

Hoffmann, P., R. Eder, T.J. Boeld, K. Doser, B. Piseshka, R. Andreesen, and M. Edinger. 2006. Only the CD45RA+ subpopulation of CD4+CD25high T cells gives rise to homogeneous regulatory T-cell lines upon in vitro expansion. Blood 108:4260-4267.

Howie, D., S.P. Cobbold, E. Adams, A. Ten Bokum, A.S. Necula, W. Zhang, H. Huang, D.J. Roberts, B. Thomas, S.S. Hester, D.J. Vaux, A.G. Betz, and H. Waldmann. 2017. Foxp3 drives 
oxidative phosphorylation and protection from lipotoxicity. JCI Insight 10.1172/jci.insight.89160.

Kamada, T., Y. Togashi, C. Tay, D. Ha, A. Sasaki, Y. Nakamura, E. Sato, S. Fukuoka, Y. Tada, A. Tanaka, H. Morikawa, A. Kawazoe, T. Kinoshita, K. Shitara, S. Sakaguchi, and H. Nishikawa. 2019. PD-1(+) regulatory T cells amplified by PD-1 blockade promote hyperprogression of cancer. Proceedings of the National Academy of Sciences of the United States of America 116:9999-10008.

Kawalekar, O.U., R.S. O'Connor, J.A. Fraietta, L. Guo, S.E. McGettigan, A.D. Posey, Jr., P.R. Patel, S. Guedan, J. Scholler, B. Keith, N.W. Snyder, I.A. Blair, M.C. Milone, and C.H. June. 2016. Distinct Signaling of Coreceptors Regulates Specific Metabolism Pathways and Impacts Memory Development in CAR T Cells. Immunity 44:380-390.

Levine, A.G., A. Arvey, W. Jin, and A.Y. Rudensky. 2014. Continuous requirement for the TCR in regulatory T cell function. Nature immunology 15:1070-1078.

Liu, X., Y. Wang, H. Lu, J. Li, X. Yan, M. Xiao, J. Hao, A. Alekseev, H. Khong, T. Chen, R. Huang, J. Wu, Q. Zhao, Q. Wu, S. Xu, X. Wang, W. Jin, S. Yu, Y. Wang, L. Wei, A. Wang, B. Zhong, L. Ni, X. Liu, R. Nurieva, L. Ye, Q. Tian, X.W. Bian, and C. Dong. 2019. Genome-wide analysis identifies NR4A1 as a key mediator of T cell dysfunction. Nature 567:525-529.

Long, A.H., W.M. Haso, J.F. Shern, K.M. Wanhainen, M. Murgai, M. Ingaramo, J.P. Smith, A.J. Walker, M.E. Kohler, V.R. Venkateshwara, R.N. Kaplan, G.H. Patterson, T.J. Fry, R.J. Orentas, and C.L. Mackall. 2015. 4-1BB costimulation ameliorates T cell exhaustion induced by tonic signaling of chimeric antigen receptors. Nature medicine 21:581-590.

Lynn, R.C., E.W. Weber, E. Sotillo, D. Gennert, P. Xu, Z. Good, H. Anbunathan, J. Lattin, R. Jones, V. Tieu, S. Nagaraja, J. Granja, C.F.A. de Bourcy, R. Majzner, A.T. Satpathy, S.R. Quake, M. Monje, H.Y. Chang, and C.L. Mackall. 2019. c-Jun overexpression in CAR T cells induces exhaustion resistance. Nature 576:293-300.

MacDonald, K.N., S. Ivison, K.L. Hippen, R.E. Hoeppli, M. Hall, G. Zheng, I.E. Dijke, M.A. Aklabi, D.H. Freed, I. Rebeyka, S. Gandhi, L.J. West, J.M. Piret, B.R. Blazar, and M.K. Levings. 2019a. Cryopreservation timing is a critical process parameter in a thymic regulatory T-cell therapy manufacturing protocol. Cytotherapy 21:1216-1233.

MacDonald, K.N., J.M. Piret, and M.K. Levings. 2019b. Methods to manufacture regulatory T cells for cell therapy. Clinical and experimental immunology 197:52-63.

Maj, T., W. Wang, J. Crespo, H. Zhang, W. Wang, S. Wei, L. Zhao, L. Vatan, I. Shao, W. Szeliga, C. Lyssiotis, J.R. Liu, I. Kryczek, and W. Zou. 2017. Oxidative stress controls regulatory $\mathrm{T}$ cell apoptosis and suppressor activity and PD-L1-blockade resistance in tumor. Nature immunology 18:1332-1341. 
McKinney, E.F., and K.G.C. Smith. 2018. Metabolic exhaustion in infection, cancer and autoimmunity. Nature immunology 19:213-221.

Mestermann, K., T. Giavridis, J. Weber, J. Rydzek, S. Frenz, T. Nerreter, A. Mades, M. Sadelain, H. Einsele, and M. Hudecek. 2019. The tyrosine kinase inhibitor dasatinib acts as a pharmacologic on/off switch for CAR T cells. Sci Transl Med 11:(499):eaau5907.

Miller, B.C., D.R. Sen, R. Al Abosy, K. Bi, Y.V. Virkud, M.W. LaFleur, K.B. Yates, A. Lako, K. Felt, G.S. Naik, M. Manos, E. Gjini, J.R. Kuchroo, J.J. Ishizuka, J.L. Collier, G.K. Griffin, S. Maleri, D.E. Comstock, S.A. Weiss, F.D. Brown, A. Panda, M.D. Zimmer, R.T. Manguso, F.S. Hodi, S.J. Rodig, A.H. Sharpe, and W.N. Haining. 2019. Subsets of exhausted CD8(+) T cells differentially mediate tumor control and respond to checkpoint blockade. Nature immunology 20:326-336.

Olesin, E., R. Nayar, P. Saikumar-Lakshmi, and L.J. Berg. 2018. The Transcription Factor Runx2 Is Required for Long-Term Persistence of Antiviral CD8(+) Memory T Cells. Immunohorizons 2:251-261.

Opstelten, R., S. de Kivit, M.C. Slot, M. van den Biggelaar, D. Iwaszkiewicz-Grześ, M. Gliwiński, A.M. Scott, B. Blom, P. Trzonkowski, J. Borst, E. Cuadrado, and D. Amsen. 2020. GPA33: A Marker to Identify Stable Human Regulatory T Cells. The Journal of Immunology 204:ji1901250.

Pacella, I., and S. Piconese. 2019. Immunometabolic Checkpoints of Treg Dynamics: Adaptation to Microenvironmental Opportunities and Challenges. Frontiers in immunology 10.3389/fimmu.2019.01889.

Saeidi, A., K. Zandi, Y.Y. Cheok, H. Saeidi, W.F. Wong, C.Y.Q. Lee, H.C. Cheong, Y.K. Yong, M. Larsson, and E.M. Shankar. 2018. T-Cell Exhaustion in Chronic Infections: Reversing the State of Exhaustion and Reinvigorating Optimal Protective Immune Responses. Frontiers in immunology 9:2569.

Saleh, R., and E. Elkord. 2019. Treg-mediated acquired resistance to immune checkpoint inhibitors. Cancer Lett 457:168-179.

Saravia, J., H. Zeng, Y. Dhungana, D. Bastardo Blanco, T.-L.M. Nguyen, N.M. Chapman, Y. Wang, A. Kanneganti, S. Liu, J.L. Raynor, P. Vogel, G. Neale, P. Carmeliet, and H. Chi. 2020. Homeostasis and transitional activation of regulatory T cells require c-Myc. Science advances 10.1126/sciadv.aaw6443.

Schlaphoff, V., S. Lunemann, P.V. Suneetha, J. Jaroszewicz, J. Grabowski, J. Dietz, F. Helfritz, H. Bektas, C. Sarrazin, M.P. Manns, M. Cornberg, and H. Wedemeyer. 2011. Dual function of the NK cell receptor 2B4 (CD244) in the regulation of HCV-specific CD8+ T cells. PLoS Pathog 7:e1002045. 
Schurich, A., L.J. Pallett, D. Jajbhay, J. Wijngaarden, I. Otano, U.S. Gill, N. Hansi, P.T. Kennedy, E. Nastouli, R. Gilson, C. Frezza, S.M. Henson, and M.K. Maini. 2016. Distinct Metabolic Requirements of Exhausted and Functional Virus-Specific CD8 T Cells in the Same Host. Cell reports 16:1243-1252.

Shahbaz, S., G. Dunsmore, P. Koleva, L. Xu, S. Houston, and S. Elahi. 2020. Galectin-9 and VISTA Expression Define Terminally Exhausted T Cells in HIV-1 Infection. J Immunol 204:2474-2491.

Tanaka, A., and S. Sakaguchi. 2019. Targeting Treg cells in cancer immunotherapy. European journal of immunology 49:1140-1146.

Thorp, E.B., C. Stehlik, and M.J. Ansari. 2015. T-cell exhaustion in allograft rejection and tolerance. Current opinion in organ transplantation 20:37-42.

Vahl, J.C., C. Drees, K. Heger, S. Heink, J.C. Fischer, J. Nedjic, N. Ohkura, H. Morikawa, H. Poeck, S. Schallenberg, D. Riess, M.Y. Hein, T. Buch, B. Polic, A. Schonle, R. Zeiser, A. Schmitt-Graff, K. Kretschmer, L. Klein, T. Korn, S. Sakaguchi, and M. Schmidt-Supprian. 2014. Continuous $\mathrm{T}$ cell receptor signals maintain a functional regulatory $\mathrm{T}$ cell pool. Immunity 41:722-736.

van der Windt, G.J., B. Everts, C.H. Chang, J.D. Curtis, T.C. Freitas, E. Amiel, E.J. Pearce, and E.L. Pearce. 2012. Mitochondrial respiratory capacity is a critical regulator of CD8+ T cell memory development. Immunity 36:68-78.

Wang, R., C.P. Dillon, L.Z. Shi, S. Milasta, R. Carter, D. Finkelstein, L.L. McCormick, P. Fitzgerald, H. Chi, J. Munger, and D.R. Green. 2011. The transcription factor Myc controls metabolic reprogramming upon T lymphocyte activation. Immunity 35:871-882.

Wang, Y., J. Hu, Y. Li, M. Xiao, H. Wang, Q. Tian, Z. Li, J. Tang, L. Hu, Y. Tan, X. Zhou, R. He, Y. Wu, L. Ye, Z. Yin, Q. Huang, and L. Xu. 2019. The Transcription Factor TCF1 Preserves the Effector Function of Exhausted CD8 T Cells During Chronic Viral Infection. Frontiers in immunology 10:169.

Weber, E.W., R.C. Lynn, K.R. Parker, H. Anbunathan, J. Lattin, E. Sotillo, Z. Good, M. Malipatlolla, P. Xu, P. Vandris, R.G. Majzner, Y. Qi, L.-C. Chen, A.J. Gentles, T.J. Wandless, A.T. Satpathy, H.Y. Chang, and C.L. Mackall. 2020a. Transient "rest" induces functional reinvigoration and epigenetic remodeling in exhausted CAR-T cells. bioRxiv 2020.2001.2026.920496.

Weber, E.W., R.C. Lynn, E. Sotillo, J. Lattin, P. Xu, and C.L. Mackall. 2019. Pharmacologic control of CAR-T cell function using dasatinib. Blood Adv 3:711-717.

Weber, E.W., M.V. Maus, and C.L. Mackall. 2020b. The Emerging Landscape of Immune Cell Therapies. Cell 181:46-62. 
Wei, J., L. Long, K. Yang, C. Guy, S. Shrestha, Z. Chen, C. Wu, P. Vogel, G. Neale, D.R. Green, and H. Chi. 2016. Autophagy enforces functional integrity of regulatory T cells by coupling environmental cues and metabolic homeostasis. Nature immunology 17:277-285.

Wherry, E.J. 2011. T cell exhaustion. Nature immunology 12:492-499.

Wherry, E.J., and M. Kurachi. 2015. Molecular and cellular insights into T cell exhaustion. Nature reviews. Immunology 15:486-499.

Wing, K., Y. Onishi, P. Prieto-Martin, T. Yamaguchi, M. Miyara, Z. Fehervari, T. Nomura, and S. Sakaguchi. 2008. CTLA-4 control over Foxp3+ regulatory T cell function. Science 322:271275.

Yang, K., D.B. Blanco, G. Neale, P. Vogel, J. Avila, C.B. Clish, C. Wu, S. Shrestha, S. Rankin, L. Long, A. Kc, and H. Chi. 2017. Homeostatic control of metabolic and functional fitness of Treg cells by LKB1 signalling. Nature 548:602-606.

Zhang, Z., S. Liu, B. Zhang, L. Qiao, Y. Zhang, and Y. Zhang. 2020. T Cell Dysfunction and Exhaustion in Cancer. Frontiers in cell and developmental biology 8:17.

Zou, D., Y. Dai, X. Zhang, G. Wang, X. Xiao, P. Jia, X.C. Li, Z. Guo, and W. Chen. 2020. T cell exhaustion is associated with antigen abundance and promotes transplant acceptance. American journal of transplantation 10.1111/ajt.15870. 
A
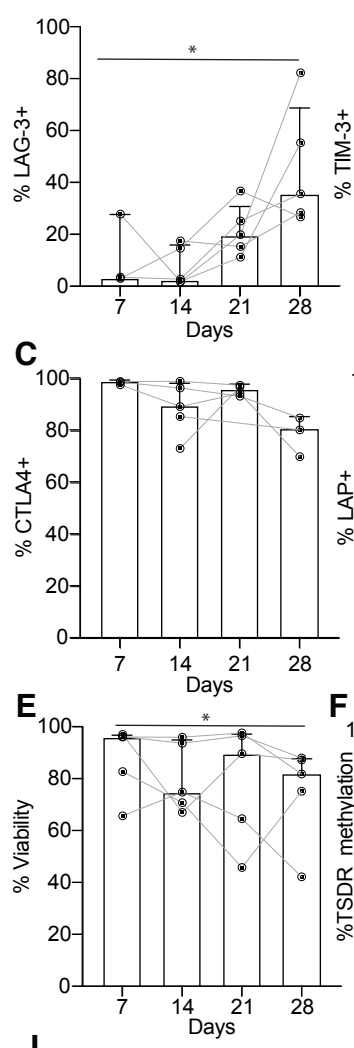

I

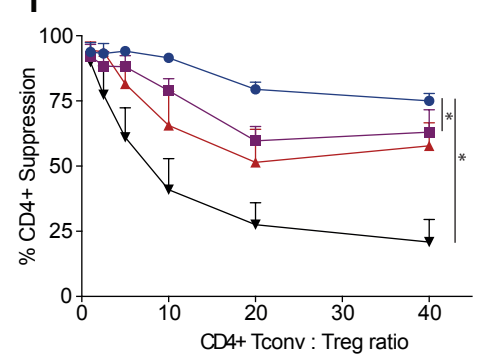

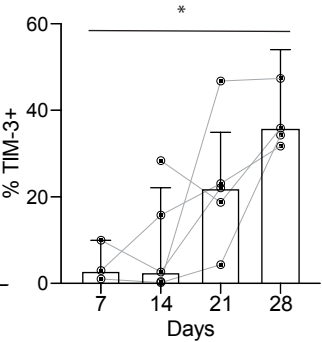
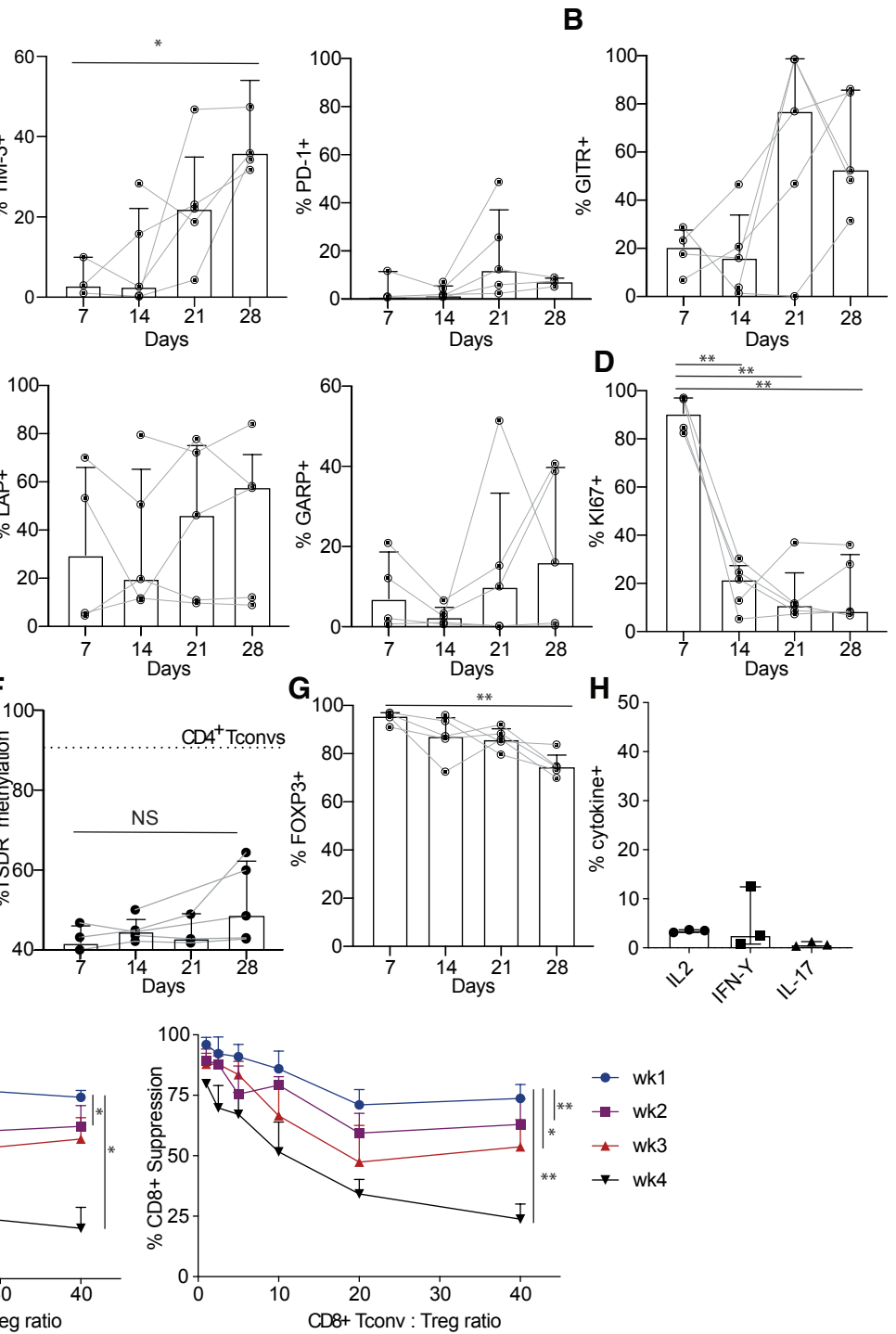

Figure 1. Repetitive stimulation of Tregs leads to dysfunction. Naïve Tregs were sorted and stimulated weekly for 4 weeks. Analysis for (A) LAG-3, TIM-3, PD-1, (B) GITR, (C) CTLA4, LAP, and GARP, (D) Ki67 and (E) viability were assessed weekly. (F) DNA was isolated and pyrosequencing was used to measure methylation status of $7 \mathrm{CpGs}$ in the Treg specific demethylation region (TSDR), only females were used for these experiments. For reference, the average amount of methylation in $\mathrm{CD}^{+}$conventional T cells (Tconv) is indicated by the dotted line. (G) Expression of FOXP3 was assessed weekly. (H) Intracellular cytokine staining was done after 28 days of culture. $\mathrm{n}=3$ from 2 independent experiments (I) Cell-proliferation dye (CPD)450-labelled PBMCs were stimulated with anti-CD3/28 Dynabeads in the absence or presence of the indicated ratio of Tregs at the end of each weekly re-stimulation. After 4 days, the division index of gated $\mathrm{CD} 4^{+}$or $\mathrm{CD}^{+} \mathrm{T}$ cells was determined and percent suppression was calculated. To compare suppression over time, suppression assays used the same responder PBMC using replicate cryopreserved aliquots. (A-H) Mixed-effects model analyses were done with Dunnett's multiple comparisons test to compare changes overtime to day 7. Each dot represents a unique donor. Median \pm interquartile range, $n=3-5$ from 3 independent experiments. (I) One-way ANOVA with Dunnett's multiple comparisons test, mean \pm SEM, $\mathrm{n}=4-5$ from 2-3 independent experiments. ${ }^{* *} \mathrm{p} \leq 0.01,{ }^{*} \mathrm{p} \leq 0.05$. 
A
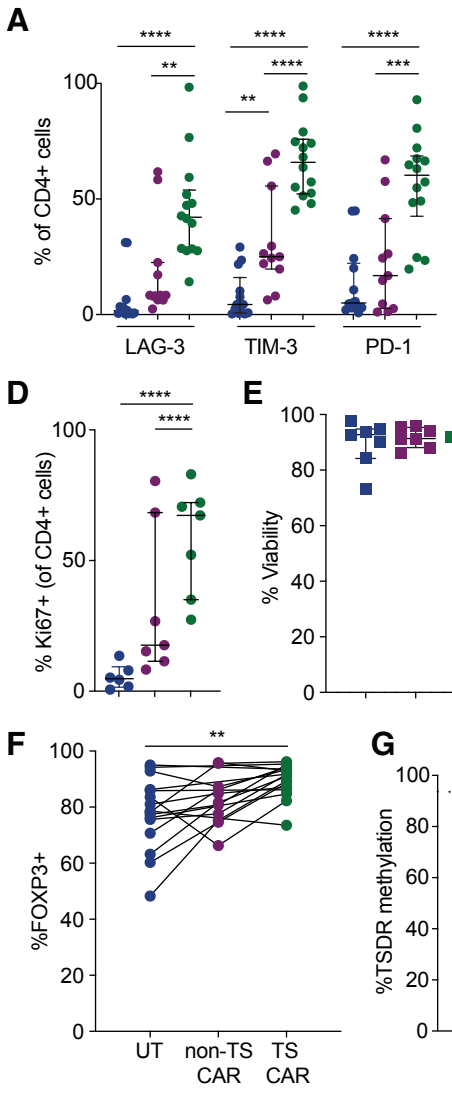
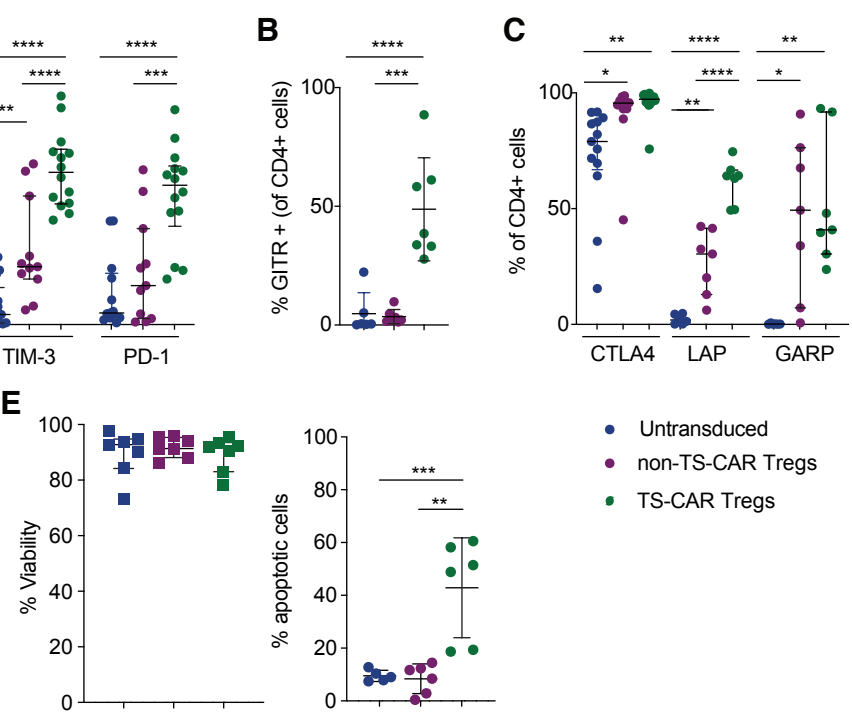

- Untransduced

- non-TS-CAR Tregs

- TS-CAR Tregs
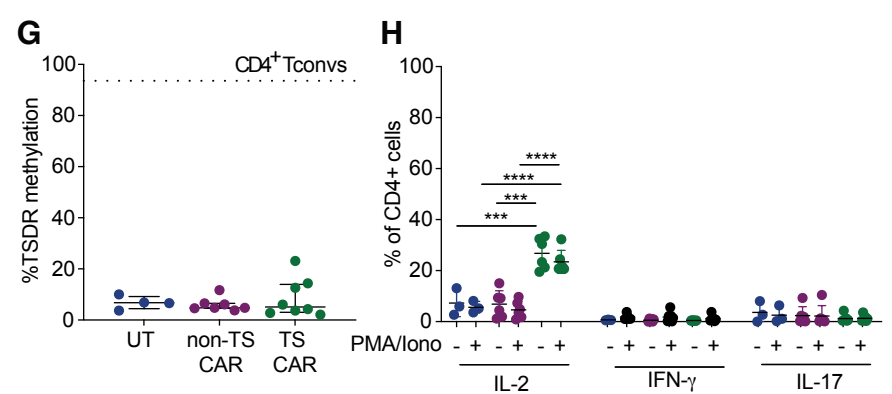

Figure 2. Expression of a tonic signaling CAR in Tregs induces phenotypic changes without lineage destabilization. Naïve Tregs were left transduced (UT) or transduced with retrovirus encoding a non-tonic signaling (non-TS) or a tonic signaling (TS-) CAR. After 11-12 days of culture cells were analyzed for expression of: (A) LAG-3, TIM-3, PD-1, (B) GITR, (C) CTLA4, LAP, GARP, and (D) Ki67. (E) Viability was assess using an automated cell counter and apoptosis by flow cytometry and (F) FOXP3 by intracellular staining. (G) DNA was isolated and pyrosequencing was used to measure methylation status of $7 \mathrm{CpGs}$ in the Treg specific demethylation region (TSDR). Data are the average methylation of the $7 \mathrm{CpGs}$. Only males were used for these experiments. For reference, the average amount of methylation in $\mathrm{CD}^{+}$ conventional T cells (Tconv) is indicated by the dotted line. (H) Intracellular cytokine production was assessed by flow cytometry 4 hours after a PMA/Ionomycin stimulation. Each dot represents a unique donor. Median \pm interquartile range. (A-H) One-way Anova with Turkey's comparisons test. For A-E (left), n=11-14 from 5-6 independent experiments. For the apoptosis assay (E), right, $\mathrm{n}=5-6$ from 3-4 independent experiments. For $\mathbf{F}, \mathrm{n}=11-17$ from 5-7 independent experiments. For $\mathbf{G}, \mathbf{n}=3-6$ from 3-4 independent experiments. For $\mathbf{H}, \mathrm{n}=3-6$ from 3 independent experiments. $* * * * \mathrm{p} \leq 0.0001, * * * \mathrm{p} \leq 0.001, * * \mathrm{p} \leq 0.01, * \mathrm{p} \leq 0.05$. 
bioRxiv preprint doi: https://doi.org/10.1101/2020.06.27.175158; this version posted June 28, 2020. The copyright holder for this preprint (which was not certified by peer review) is the author/funder, who has granted bioRxiv a license to display the preprint in perpetuity. It is made available under aCC-BY-NC-ND 4.0 International license.

A

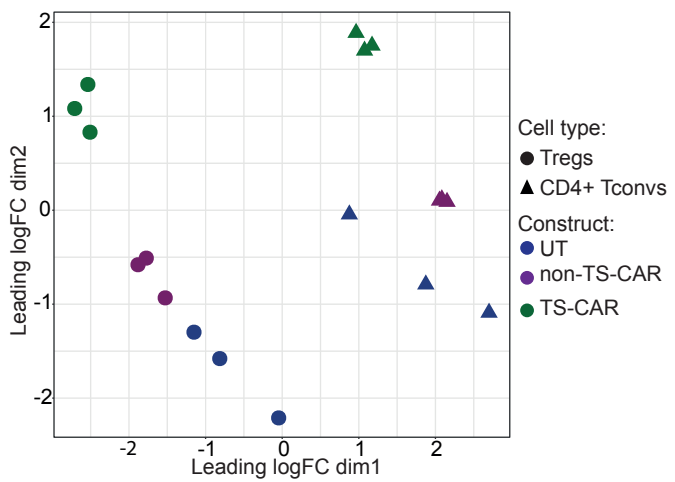

C

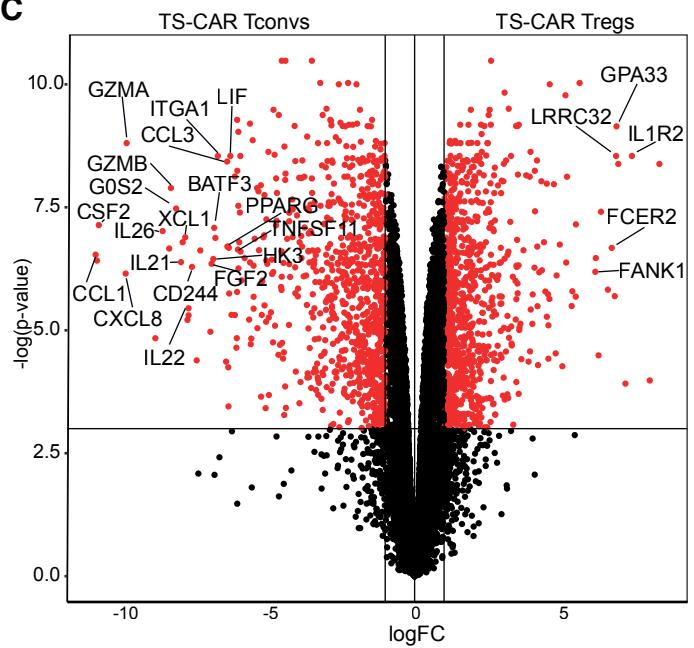

E TS CAR Tregs Vs UT and non-TS CAR Tregs
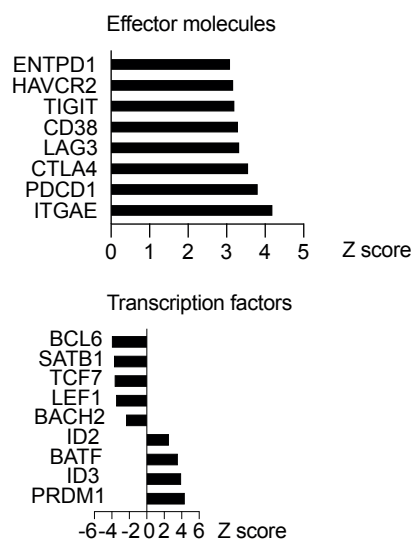

B TS-CAR Tregs vs TS-CAR CD4+ Tconvs

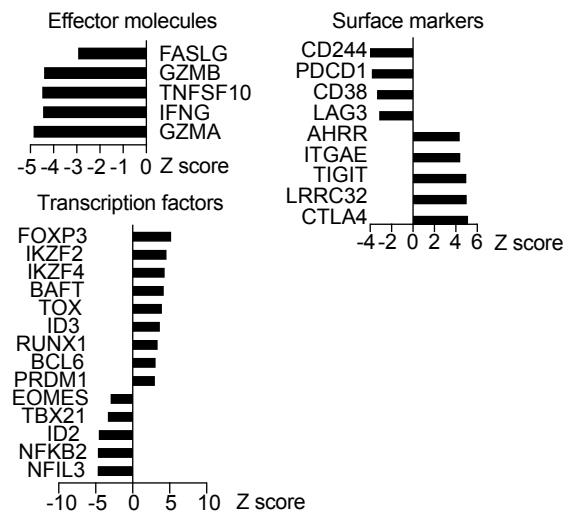

D

TS-CAR Tregs $\square$ non-TS-CAR Tregs $\square$ UT Tregs

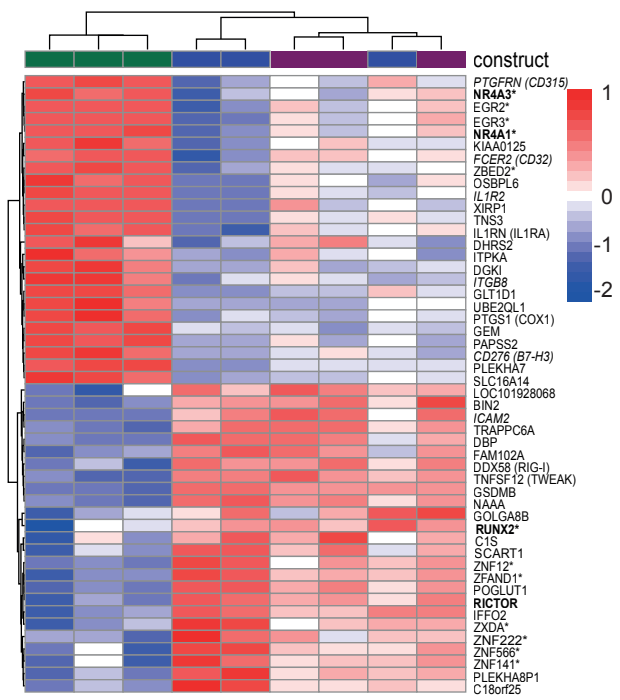

$\mathbf{F}$

Hallmark pathways analysis upregulated in TS-CAR Tregs vs UT and non-TS-CAR Tregs

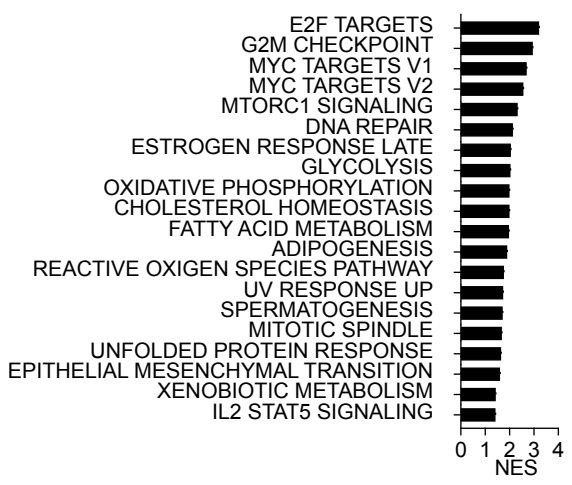

Figure 3. Expression of a tonic signaling CAR results in differential effects on the transcriptome of Tregs and Tconvs. After 12 days of culture, RNA was extracted from Tregs or $\mathrm{CD} 4^{+}$Tconvs that were untransduced (UT) or transduced to express a non-tonic signaling (nonTS) or a tonic signaling (TS-) CAR, and subject to RNA sequencing. (A) Multidimensional 
scaling plots comparing the transcriptome of the indicated types of Tregs and Tconvs. (B) Z score of differentially expressed genes in TS-CAR Tregs compared to UT or non-TS-CAR Tregs. Threshold used was a $\operatorname{logFC}>1$ and adj.pvalue $<0.05$, and $\log \mathrm{FC}<-1$ and adj.pvalue $<$ 0.05. (C) Volcano plot comparing the gene expression profile of TS-CAR Tregs versus TS-CAR Tconvs after removal of genes differentially expressed (upregulated and downregulated) between UT Tconvs and UT Tregs (see Supp. Figure 3A). Genes in red are defined by a p-value $<0.05$. (D) Heatmap showing the top 50 differentially expressed genes between the different types of Tregs. Bold genes are associated with exhaustion in conventional $\mathrm{T}$ cells, italicized genes are cell surface proteins and those marked with * are transcription factors. (E) Z score comparing genes that were significatively upregulated or downregulated in TS-CAR Tregs versus TS-CAR Tconvs. Threshold used was a $\log \mathrm{FC}>1$ and adj.pvalue $<0.05$, and $\operatorname{logFC}<-1$ and adj.pvalue $<$ 0.05. (F) Gene set enrichment analysis. Normalized enrichment score (NES) of hallmark pathways analysis overrepresented in TS-CAR Tregs compared to non-TS CAR or UT Tregs cells. $\mathrm{n}=3$ per group from 2 independent experiments. 

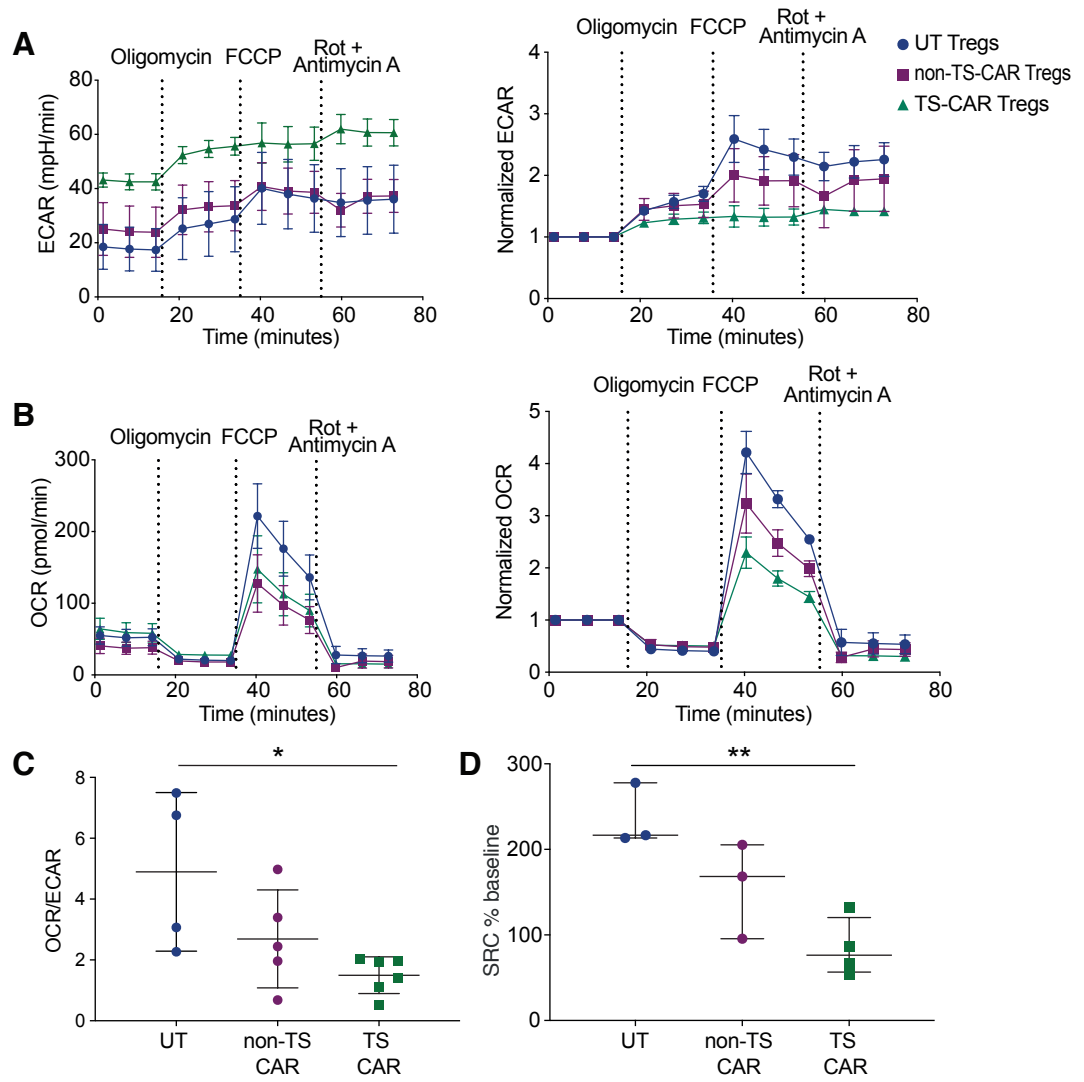

Figure 4. Expression of a tonic signaling CAR signaling alters Treg metabolism. Tregs were untransduced (UT) or transduced to express a non-tonic signaling (non-TS) or a tonic signaling (TS-) CAR. After 11-12 days of culture cells were plated in Cell-Tak coated wells and subject to the Agilent Seahorse XF Cell Mito Stress Test. (A) Extracellular acidification rate (ECAR) (left) and normalized to baseline ECAR (right) over time, (B) Oxygen consumption rate (OCR) (left) and normalized to baseline OCR (right) over time. Oligomycin, FCCP and a mix of Rotenone and antimycin A were added as indicated. A\&B) n=3-5 donor from 2 independent experiments, using the average of technical replicates. Mean \pm SEM. (C) OCR/ECAR ratio at baseline. Oneway Anova with Turkey's comparisons test $\mathrm{p}=0.0196$. $\mathrm{n}=4-6$ from 3 independent experiments. (D) The spare respiratory capacity (SRC) was calculated by the average of the maximum OCR after FCCP injection minus the average basal respiration, divided by the average basal respiration, times 100 ((max-basal)/basal*100). One-way Anova with Turkey's comparisons test $\mathrm{p}=0.0054, \mathrm{n}=3-4$ from 2 independent experiments. 

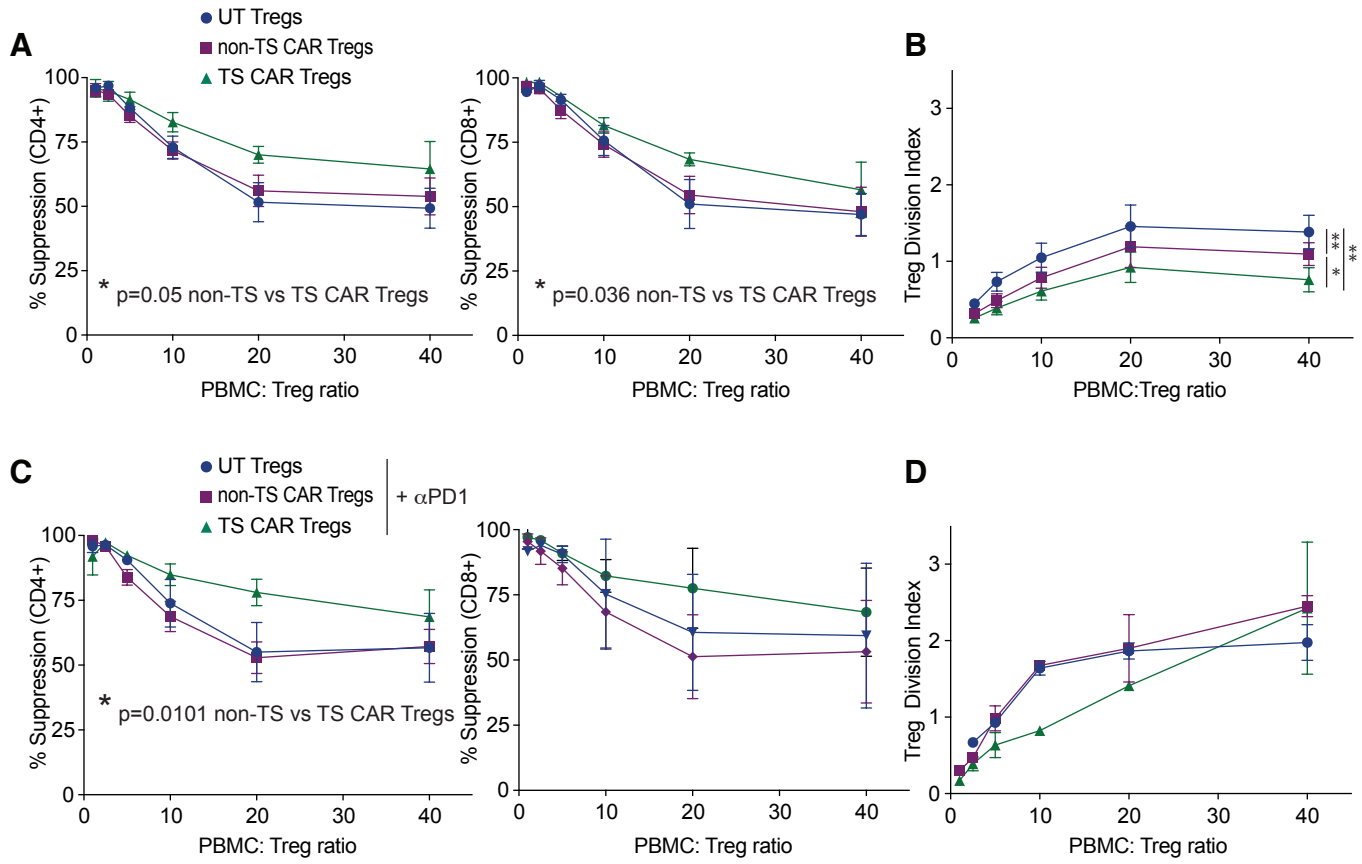

D

E
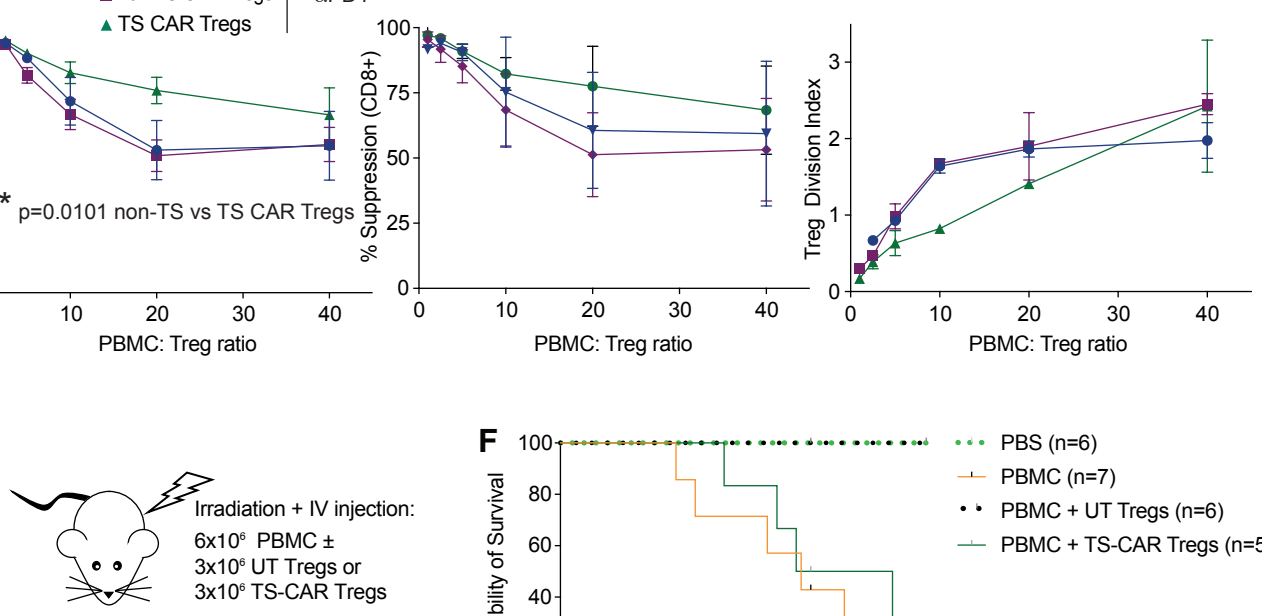

NSG mice
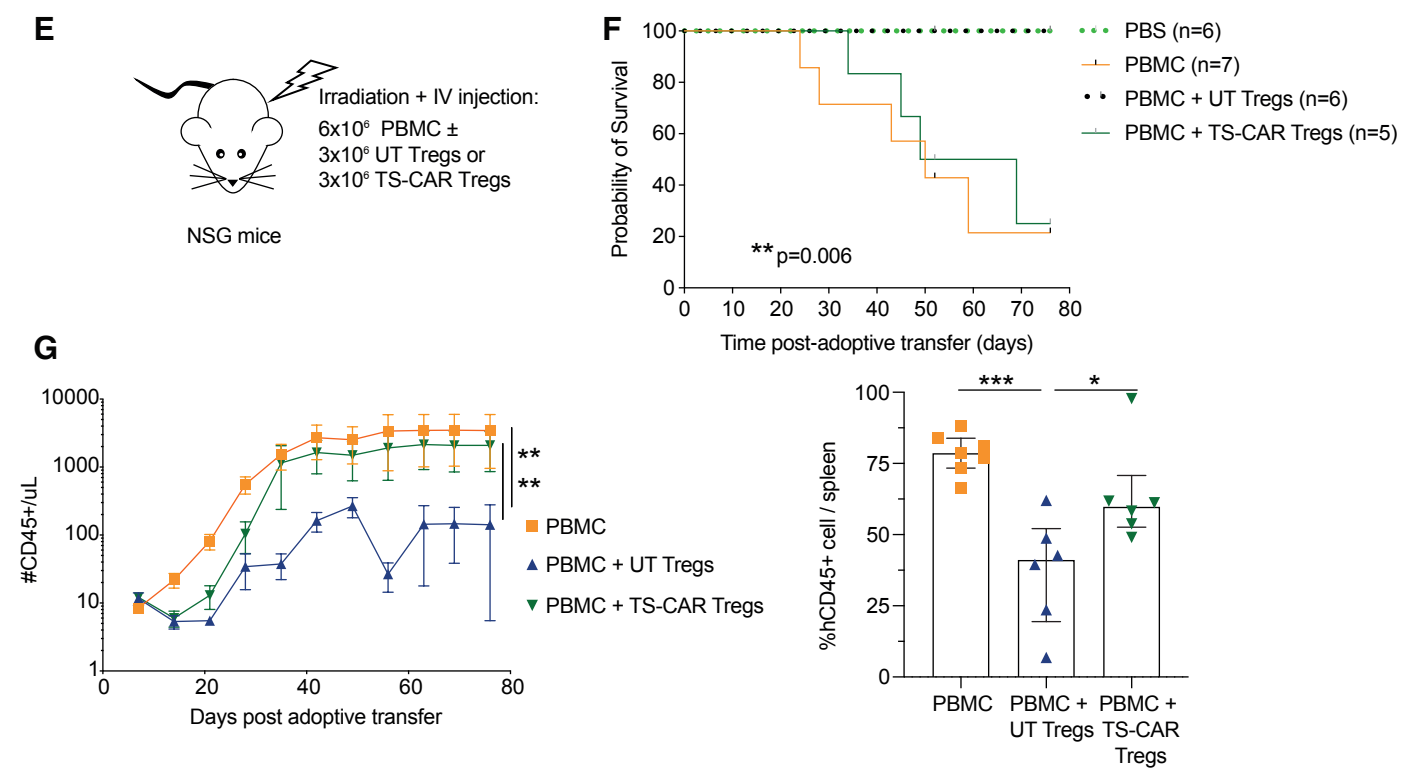

Figure 5. Tonic signaling CAR Tregs are functional in vitro but not in vivo. (A) Suppression of CPD-ef450-labeled PBMCs by untransduced (UT), non-tonic signaling (non-TS) or tonic signaling (TS-) CAR CPD-ef-670-labeled Tregs was determined after 4 days of co-culture. $n=8$ 10 from 4 independent experiments (B) The Treg division index from cultures in (A) was determined. $n=6-7$ from 3 independent experiments (C \&D) Suppression assays were done with the addition of nivolumab (anti-PD-1 mAb) at 10 $\mathrm{mg} / \mathrm{ml}$. (A-D) Analysis were done using oneway ANOVA with Turkey's multiple comparisons test. (E-G) Irradiated NSG mice were injected with PBS or $6 \times 10^{6}$ PBMCs without or with $3 \times 10^{6}$ UT or TS-CAR Tregs. Data from 2 independent experiments (E) Schematic diagram of the experiment set up. (F) Survival curve, log-rank (Mantel-Cox) test. (G) Absolute number of human CD45+ cells/ $\mu \mathrm{L}$ of blood assessed 
weekly after adoptive transfer. One-way ANOVA with Dunnett's multiple comparisons test (left) and \% Human CD45+ engraftment in the spleen upon experimental or humane endpoint (of live singlets) in the different groups (right). One-way ANOVA with Turkey's multiple comparisons test. 